\title{
Circular Dichroism in Core Level Photoemission from an Adsorbed Chiral Molecule
}

\author{
M. Polcik, ${ }^{1}$ F. Allegretti, ${ }^{2}$ D. I. Sayago, ${ }^{1}$ G. Nisbet,${ }^{3}$ C. L. A. Lamont,${ }^{3}$ and D. P. Woodruff ${ }^{2}$ \\ ${ }^{1}$ Fritz-Haber-Institut der Max-Planck-Gesellschaft, Faradayweg 4-6, D-14195 Berlin, Germany \\ ${ }^{2}$ Physics Department, University of Warwick, Coventry CV4 7AL, United Kingdom \\ ${ }^{3}$ Centre for Applied Catalysis, Department of Chemical and Biological Sciences, University of Huddersfield, Queensgate, \\ Huddersfield HDI 3DH, United Kingdom
}

(Received 9 February 2004; published 10 June 2004)

\begin{abstract}
The results of experimental measurements and theoretical simulations of circular dichroism in the angular dependence (CDAD) of photoemission from atomic core levels of each of the enantiomers of a chiral molecule, alanine, adsorbed on $\mathrm{Cu}(110)$ are presented. Measurements in, and out of, substrate mirror planes distinguish CDAD due to the chirality of the sample and the experimental geometry. The effect due to sample chirality is relatively weak, so such measurements may not provide a routine spectral fingerprint of adsorbate chirality.
\end{abstract}

DOI: 10.1103/PhysRevLett.92.236103

PACS numbers: 68.43.Fg, 33.55. Ad, 79.60.Dp

There has been growing interest in the last few years in the properties of chiral molecules adsorbed on surfaces (e.g., [1-6]), motivated in part by the potential importance of producing molecular products of a single "handedness" (enantioselective) by heterogeneous catalysis. In such studies a simple spectroscopic measurement, which could establish if the surface species (which may be unknown reaction intermediates) are predominantly of a single chirality, would be of great value. Conventional optical absorption measurements on submonolayer coverages of adsorbed molecules which exploit the circular dichroism (a difference in absorption for left- and rightcircularly polarized radiation) are unlikely to be fruitful. However, spatially oriented chiral molecules, such as those adsorbed on a surface, should show circular dichroism in the angular distribution (CDAD) of photoelectrons emitted from these species [7,8]. Indeed, such an effect has recently been observed [9] even for randomly oriented chiral molecules in the gas phase. Here we present the results of experiments, and model calculations, which evaluate this idea using core level photoemission from one model system, alanine on $\mathrm{Cu}(110)$; our results confirm the existence of the effect but show it is weak relative to other sources of CDAD unrelated to the surface chirality, casting doubt on the likely utility of this phenomenon to provide a routine spectral fingerprint.

We chose core level photoemission for these CDAD studies because the elemental and chemical-state specificity offered by photoelectron binding energies allows one to localize the information on chirality to specific adsorbed species on a surface; this is not true for photoemission from the valence states in which contributions from substrate and coexistent adsorbed species generally overlap. An important aspect of CDAD, however, is that even nonchiral surfaces give rise to a significant signal if the geometry of the experiment is chiral [10]. If the plane defined by the incident (circularly polarized) light and the photoelectron collection direction does not corre- spond to a mirror symmetry plane of the sample, the experiments conducted with opposite circular polarizations will not be equivalent and so a circular dichroism signal will be observed. This CDAD effect (up to $50 \%$ or more) from core levels of atoms in nonchiral surfaces is well established from substrate atoms [e.g., $\mathrm{Si}(100)$ [11,12] and $\mathrm{W}(110)$ [13] ], from adsorbed atoms (e.g., Rb and $\mathrm{K}$ [14]), and from adsorbed linear molecules (CO [15-17] and NO [18]). A key question which we aim to answer is this: Can a CDAD effect due to adsorbate chirality clearly be distinguished from that due to these other effects?

CDAD in core level photoemission is closely related to the phenomenon of photoelectron diffraction, the measured angular distribution of the photoelectrons and the circular dichroism in this signal, being determined by the elastic scattering of the atoms surrounding the emitter. Through our use of scanned-energy mode photoelectron diffraction $(\mathrm{PhD})$ for surface structure determination, we have well proven multiple scattering computer codes [19] to simulate this effect, and inclusion in these codes of circularly polarized incident radiation has allowed us to calculate the associated core level CDAD. As a check on the integrity of this code we have performed simulations of previously published experimental measurements of the $\mathrm{C} 1 s$ and $\mathrm{O} 1 s$ photoemission from $\mathrm{CO}$ on $\mathrm{Pd}(111)$ [16] for which the adsorption geometry is known [20] and there are thus no adjustable parameters. Figure $1 \mathrm{com}-$ pares the experimental data and theoretical simulations of the polar emission angle dependence of the CDAD from $\mathrm{C} 1 s$ at two different photon energies and $\mathrm{O} 1 s$ (the nominal photoelectron kinetic energies are given in the figure). In these experiments the detector was scanned in a plane perpendicular to the surface and perpendicular to the plane defined by the surface normal and the incident radiation. Our calculations reproduce the experimental results well both in the angular dependence and the amplitude of the CDAD, which is much weaker in the 


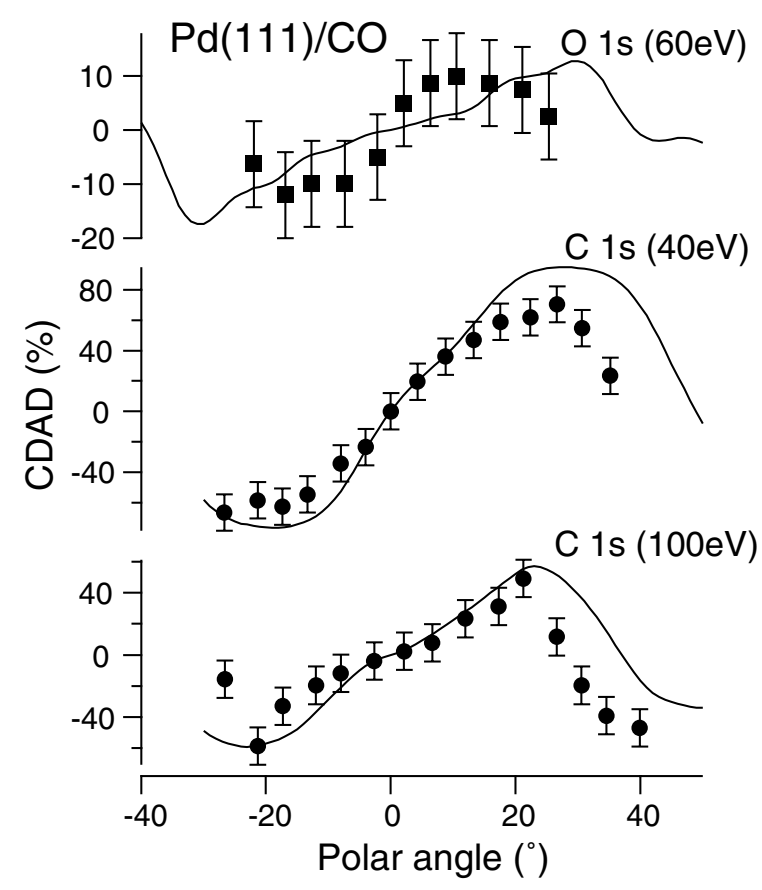

FIG. 1. Comparison of the measured (individual points) CDAD from $\mathrm{CO}$ on $\mathrm{Pd}(111)$ [16] with the results of our simulations (full lines). The CDAD is shown, for both $\mathrm{O} 1 \mathrm{~s}$ and $\mathrm{C} 1 \mathrm{~s}$ photoemission at different photoelectron kinetic energies, as a function of polar emission angle in a plane perpendicular to the incidence plane.

O $1 s$ signal than in the C $1 s$ signal. Notice that the dichroism is zero at normal emission for all the measurements, because under this condition the experimental geometry, as well as the surface structure, is nonchiral.

As a model system we have studied alanine, $\mathrm{NH}_{2} \mathrm{CH}_{3} \mathrm{C}^{*} \mathrm{HCOOH}$, the simplest chiral amino acid, having a chiral center at the $\mathrm{C}^{*}$ atom (the so-called $\alpha$-C) which is bonded to an $\mathrm{NH}_{2}$ amino, $\mathrm{CH}_{3}$ methyl, atomic $\mathrm{H}$, and a $\mathrm{COOH}$ carboxylic acid group. Photoemission from the $1 s$ state of these three chemically inequivalent $\mathrm{C}$ atoms in the adsorbed species can be distinguished by chemical shifts in the photoelectron binding energy, as shown in Fig. 2, the peaks being identified by comparison with spectra from glycine, formic acid, and acetic acid on $\mathrm{Cu}(110)$ [21]. On adsorption on $\mathrm{Cu}(110)$ the acid hydrogen atom is lost to form alaninate, $\mathrm{NH}_{2} \mathrm{CH}_{3} \mathrm{C}^{*} \mathrm{HCOO}-$, in several ordered phases including the nominal $(3 \times 2)$ phase $[4-6,22]$ studied here. The local adsorption geometry is believed, on the basis of STM images [6] and a PhD study [23], to be similar to that of the simpler (nonchiral) amino acid glycine $\left(\mathrm{NH}_{2} \mathrm{CH}_{2} \mathrm{COOH}\right)$ which has been studied extensively on $\mathrm{Cu}(110)$ [24-28]. Bonding to the surface is through the amino group (with the $\mathrm{N}$ near atop a surface $\mathrm{Cu}$ atom) and the carboxylate $\mathrm{O}$ atoms (in off-atop sites), the $\mathrm{C}$-C backbone being aligned approximately in a $\langle 001\rangle$ azimuth. The experiments were conducted in an ultrahigh vacuum surface

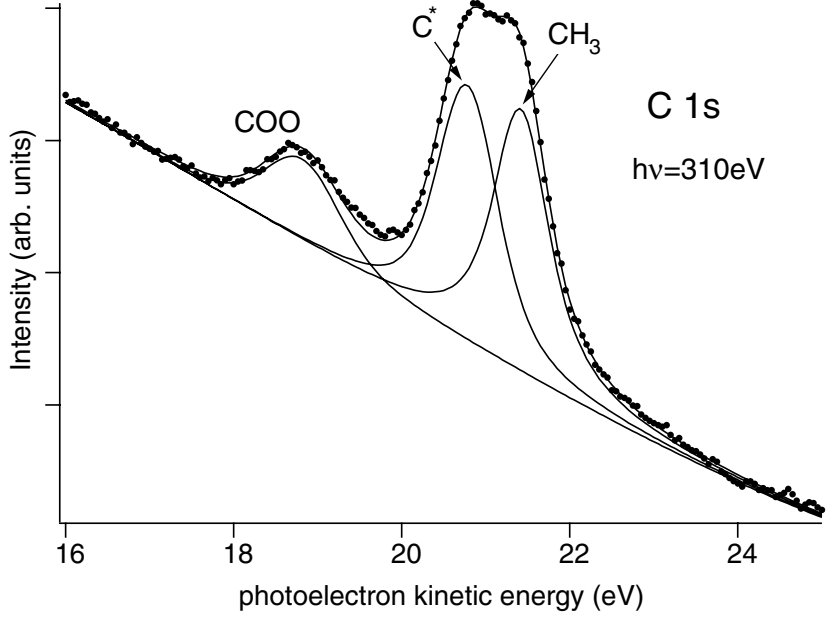

FIG. 2. Photoelectron kinetic energy spectrum obtained using a nominal photon energy of $310 \mathrm{eV}$ in the energy range of the $\mathrm{C} 1 s$ emission from alanine on $\mathrm{Cu}(110)$ showing the three distinct chemically shifted components.

science end station installed on the UE56/2-PGM-2 beam line of BESSY II which uses a $56 \mathrm{~mm}$ period undulator to deliver circularly polarized radiation [29]. The $\mathrm{Cu}(110)$ sample surface was cleaned in situ by cycles of Ar ion bombardment and annealing and characterized by LEED and by soft-x-ray photoelectron spectroscopy using the incident synchrotron radiation. The two different enantiomers of alanine (having opposite chirality), L-analine and D-analine, were contained in glass tubes which could be heated via surrounding copper coils. The sources were outgassed before dosing the $\mathrm{Cu}(110)$ crystal, at room temperature, and annealing at $470 \mathrm{~K}$ to produce the $(3 \times 2)$ phase; the coverage was estimated relative to saturation by monitoring the heights of the $\mathrm{N} 1 s, \mathrm{O} 1 s$, and $\mathrm{C} 1 s$ photoemission peaks.

For measurements in which the incident radiation, the surface normal, and the detector are all coplanar, as in our instrument, setting this plane to coincide with a mirror plane of a nonchiral surface will yield a zero CDAD signal. However, if a chiral molecule is deposited on the surface, the mirror plane is lost and a CDAD signal should appear. The mirror plane may also be lost if a nonchiral adsorbate adopts a low symmetry configuration on the surface, but in this case an experiment will average over equally probable domains related by the missing mirror plane, so no net CDAD signal will be detected. Within the substrate mirror plane, therefore, the measured CDAD is a direct consequence of the chirality of the adsorbed molecule. Because a finite CDAD signal should result even from a nonchiral surface in measurements out of the substrate mirror plane, however, we have recorded the CDAD as a function of the azimuthal rotation of the sample in order to follow the trends of the signal around the mirror planes. In this way we may expect to distinguish a true effect due to the chiral 
adsorbate, from an accidental one due to slight sample misalignment. The results of such measurements for the three components in the $\mathrm{C} 1 s$ photoemission signal from surfaces formed from each of the two enantiomers of alanine are shown in Fig. 3 for photon incidence and electron collection polar angles of $30^{\circ}$ and a photon energy of $310 \mathrm{eV}$. Notice that because of the effects of photoelectron diffraction from the three differently located inequivalent $\mathrm{C}$ emitters, the relative intensities of the three components in the C $1 s$ spectra (e.g., Fig. 2) depend strongly on emission geometry; fitting of these by three contributions with widths and energy separations constrained to common values for all such spectra is therefore rather reliable. As expected from previous measurements on nonchiral surfaces, Fig. 3 shows that there are significant CDAD signals in azimuthal angles away from the two $(\langle 110\rangle$ and $\langle 001\rangle)$ mirror planes at $0^{\circ}$ and $90^{\circ}$, the largest values of almost $30 \%$ being found for the $\alpha$-C $\left(\mathrm{C}^{*}\right)$. However, these effects are almost the same (with the same sign) for the two distinct enantiomers, showing that this effect is unrelated to the molecular chirality. In the two mirror planes of the substrate the CDAD is weak for all three C $1 s$ signals, the CDAD switching sign at azimuths close to the mirror planes in several cases. For a nonchiral surface the CDAD signal

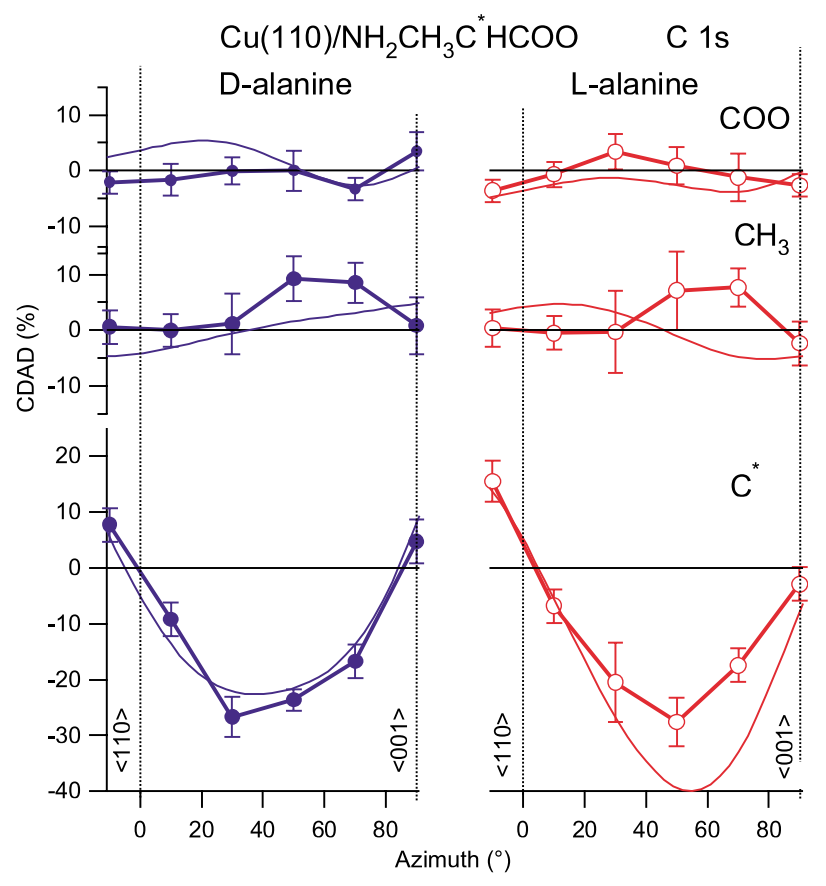

FIG. 3 (color online). $1 s$ photoemission CDAD for the three distinct chemically shifted components (Fig. 2) for D-alanine and L-alanine adsorbed on $\mathrm{Cu}(110)$ measured as a function of azimuthal angle (relative to a $\langle 110\rangle$ azimuth) at $30^{\circ}$ polar emission angle and a photon energy of $310 \mathrm{eV}$. The filled and empty circles joined by bold lines correspond to the measured values from the $\mathrm{D}$ and $\mathrm{L}$ enantiomers, respectively. The corresponding theoretical simulations are shown as thin lines. should be identically zero in these azimuths. However, in the $\langle 001\rangle$ azimuth, in particular, the CDAD is nonzero by an amount which is just statistically significant, the size of the error bars reflecting the scatter of different data sets and different methods of normalizing the signals from the two different polarizations in the data reduction. Significantly, these nonzero CDAD signals in the substrate mirror plane are equal and opposite for the two enantiomers, exactly as expected for an effect derived exclusively from the molecular chirality.

Also included in Fig. 3 as thin lines are the results of simulations for alaninate on $\mathrm{Cu}(110)$. These calculations were based on the optimized adsorption sites of the $\mathrm{N}$ and $\mathrm{O}$ atoms found in our PhD study of this surface [23] but included limited structural optimization, mainly of the internal molecular conformation with interatomic bond lengths tightly constrained to their expected values. Clearly these simulations reproduce the strong CDAD seen in the $\mathrm{C}^{*}$ emission quite well, but the detailed agreement for the much weaker CDAD of the other emitters is less good. Nevertheless, key features of the differences in the CDAD from the two enantiomers, which arise from the chirality, are reproduced as are the signs of the chirally related CDAD in the $\langle 001\rangle$ azimuth. The theoretical calculations give values for the $\mathrm{D}$ - and $\mathrm{L}$-alanine, respectively, which are $+1 \%$ and $-1 \%$ for $\mathrm{COO},+4 \%$ and $-4 \%$ for $\mathrm{CH}_{3}$, and $+8 \%$ and $-8 \%$ for $\mathrm{C}^{*}$; the corresponding experimental values are $+3 \% \pm 3 \%$ and $-3 \% \pm 2 \%,+1 \% \pm 4 \%$ and $-3 \% \pm 4 \%$, and $+3 \% \pm$ $3 \%$ and $-5 \% \pm 4 \%$.

One important feature of the results of Fig. 3 (at least in the $\langle 001\rangle$ azimuth) is that the finite equal-and-opposite CDAD in the substrate mirror planes occurs for all three $\mathrm{C}$ emitters, and not only for the $\mathrm{C}^{*}$. In the gas phase, in which one averages over random molecular orientations of the molecule, only emission from the $\mathrm{C}^{*}$ atom is expected to show local chiral effects, but on the surface this is no longer the case. When the chiral molecule is adsorbed onto the surface all the surface becomes formally chiral. Notice that no averaging over domains related by the missing mirror planes of the substrate is possible, because these would involve the different enantiomer of the adsorbate. Indeed, the magnitude of the chiral effects in the emission from the other $\mathrm{C}$ atoms in Fig. 3 may be limited by the choice of the alaninate on $\mathrm{Cu}(110)$ as a model system, because the carboxylate $\mathrm{C}$, in particular, lies close to both mirror planes of the surface and may therefore show rather weak CDAD under these conditions. The significantly larger CDAD measured in the $\langle 001\rangle$ azimuth relative to the $\langle 110\rangle$ may also be attributable to the specific geometry of the alaninate, although the theoretical calculations predict similar effects in the two substrate mirror planes.

One of the goals of this investigation was to establish whether CDAD in photoemission from the core levels of atoms in adsorbed molecular species, when measured in 
an appropriate geometry, may provide a viable spectral fingerprint of this molecular chirality. Our results show that there is indeed a measurable effect and define some of the ways in which this may be optimized. Our results also show, however, that the well-established CDAD effect due to a chiral experimental geometry is significantly larger than the effect due to the molecular chirality, at least for the example of alanine on $\mathrm{Cu}(110)$. Even on the basis of this one model system it is clear that such CDAD measurements could be used as an effective spectral fingerprint only with great care. One must either be very sure that the experimental geometry is such as to ensure that only chiral molecule effects are seen, or perform some kind of angular scans around the special experimental geometries of interest in order to distinguish the two effects. Any need to perform such angular scans increases the complexity and time scale of the experiment as a simple spectral fingerprint.

In view of this rather cautious conclusion regarding the utility of CDAD to characterize the presence of a single enantiomer of a chiral species on the surface one may consider alternative approaches. A special case arises when the adsorbed chiral molecules form a long-range ordered phase which is itself chiral; a LEED pattern then reveals the reduced symmetry directly [6]. More generally, probes of local, rather than long-range structure, offer a means of identifying the presence of chiral species on a surface. X-ray photoelectron diffraction (XPD) (exploiting high energy forward scattering) has been used to determine the orientation and aspects of the internal conformation of adsorbed chiral molecules even when there is no long-range ordering [30]. Such XPD experiments are, however, extremely time-consuming and certainly not a simple spectral fingerprint.

Of course, traditional circular dichroism experiments measure the absorption of circularly polarized radiation, and, even for submonolayer coverages of adsorbed molecules, $\mathrm{x}$-ray absorption measurements are perfectly possible by monitoring a signal related to the core-hole decay and forming the basis of NEXAFS (near-edge $\mathrm{X}$-ray absorption fine structure) which is used as a spectral fingerprint of molecular adsorbates. The possibility of using CD in NEXAFS for adsorbed chiral species (small amino acids) has been explored theoretically [31], but these calculations indicate that the effect is very small (much less than 1\%); as part of the present experiments we did conduct limited tests of CD in NEXAFS for adsorbed alanine but failed to detect any significant effect, although our investigation could not exclude effects as weak as those predicted in these theoretical calculations.

In summary, our results show that with an appropriate choice of experimental geometry a CDAD effect due specifically to the chirality of an adsorbed molecule can be detected, but the effect is weak relative to the CDAD seen in chiral experimental geometries from nonchiral surfaces. This may limit the general utility of this technique to provide a simple spectroscopic probe of adsorbate chirality in surface reactions, but it is not clear that there is any suitable alternative means of achieving this goal.

This work was supported by the Engineering and Physical Sciences Research Council (U.K.) and by the European Community through Large Scale Facilities support to BESSY.

[1] M. Ortega Lorenzo et al., Nature (London) 404, 376 (2000).

[2] M. Schunack et al., Angew. Chem., Int. Ed. Engl. 40, 2623 (2001).

[3] A. Kühlne et al., Nature (London) 415, 891 (2002).

[4] R. Raval, Catech 5, 12 (2001).

[5] Q. Chen et al., PhysChemComm 2, 9/05986E (1999).

[6] S. M. Barlow and R. Raval, Surf. Sci. Rep. 50, 201 (2003).

[7] B. Ritchie, Phys. Rev. A 12, 567 (1975); 13, 1411 (1976).

[8] N. A. Cherepkov, Chem. Phys. Lett. 87, 344 (1982).

[9] U. Hergenhahn et al., J. Chem. Phys. 120, 4553 (2004).

[10] G. Schönhense, Phys. Scr. T31, 255 (1990).

[11] H. Diamon et al., Jpn. J. Appl. Phys. 32, L1480 (1993).

[12] A. P. Kaduwela et al., Phys. Rev. B 52, 14927 (1995).

[13] R. X. Ynzunza et al., J. Electron Spectrosc. Relat. Phenom. 106, 7 (2000).

[14] A. Oelsner et al., Surf. Sci. 331-333, 349 (1995).

[15] J. Bansmann et al., Phys. Rev. B 46, 13496 (1992).

[16] J. Bansmann et al., Z. Phys. D 33, 257 (1995).

[17] C. Westphal et al., Phys. Rev. B 50, 6203 (1994).

[18] G. H. Fecher et al., J. Electron Spectrosc. Relat. Phenom. 76, 97 (1995).

[19] V. Fritzsche, J. Phys. Condens. Matter 2, 1413 (1990); Surf. Sci. 265, 187 (1992); 213, 648 (1989).

[20] T. Gie $\beta$ el et al., Surf. Sci. 406, 90 (1998).

[21] J. Hasselström et al., Surf. Sci. 407, 221 (1998).

[22] J. Williams, S. Haq, and R. Raval, Surf. Sci. 368, 303 (1996).

[23] D. I. Sayago et al. (to be published).

[24] S. M. Barlow et al., Surf. Sci. 401, 322 (1998).

[25] J. Hasselström et al., Surf. Sci. 407, 221 (1998).

[26] M. Nyberg et al., J. Chem. Phys. 112, 5420 (2000).

[27] N. A. Booth et al., Surf. Sci. 397, 258 (1998).

[28] J.-H. Kang et al., J. Chem. Phys. 118, 6059 (2003).

[29] K. J. S. Sawhney et al., Nucl. Instrum. Methods Phys. Res., Sect. A 390, 395 (1997).

[30] R. Fasel et al., J. Chem. Phys. 115, 1020 (2001).

[31] O. Plashkevych et al., Chem. Phys. 232, 49 (1998). 